\title{
Factores de riesgo de depresión post ictus isquémico
}

\author{
Risk factors for depression after and ischemic ictus \\ Mercedes Herrera ${ }^{1, a}$, Jaime Lama ${ }^{1,2, b}$, Jhony De la Cruz ${ }^{1,3, c}$

\section{RESUMEN}

Objetivo: Identificar los factores de riesgo de depresión post ictus isquémico (DPI) en pacientes adultos. Material y métodos: Estudio tipo caso y control no pareado. Se incluyeron 81 casos y 81 controles de pacientes del consultorio externo de neurología del Hospital Santa Rosa de Lima, atendidos entre setiembre y diciembre del 2018 . Se registraron variables clínicas y epidemiológicas mediante una ficha de datos. Para el diagnóstico de depresión se empleó la Hamilton Depression Rating Scale (HDRS). Se establecieron asociaciones mediante el cálculo de los Odds Ratio (OR) con intervalos de confianza del 95\% (IC). Se compararon los casos y controles usando un análisis multivariado de regresión logística. Resultados: Las variables asociadas con DPI fueron: tabaquismo (OR=4,67; IC95\%, 1,99-10,92, p<0,001), lesión localizada en el hemisferio izquierdo (OR=3,11; IC 95\%, 1,41-6,84, p=0,005) y deterioro cognitivo $(\mathrm{OR}=3,18$; IC95\%, 1,42-7,10, $\mathrm{p}=0,005)$. Conclusiones: En pacientes con antecedente de ictus isquémico, el tabaquismo, la localización de la lesión en el hemisferio cerebral izquierdo y el deterioro cognitivo, se asociaron con un mayor riesgo de depresión. Se necesitan estudios adicionales para definir mejor la naturaleza de dichas asociaciones.

PALABRAS CLAVE: Accidente cerebrovascular, factores de riesgo, depresión, disfunción cognitiva. (Fuente: DeCS Bireme).

\section{SUMMARY}

Objective: To identify risk factors for depression after and ischemic ictus (DII) in adults. Methods: A non-matched case-control study was carried-out including 81 cases and controls, respectively recruited from the neurologic outpatient clinic of Hospital Santa Rosa de Lima from September to December of 2018. Clinical and epidemiological data were gathered and stored in a data base. The Hamilton Depression Rating Scale was used to diagnose depression. Odds rations and $95 \%$ CI were calculated and a multivariate logistic regression analysis was used. Results: smoking $(\mathrm{OR}=4.67$; IC95\%, 1.99-10.92, $\mathrm{p}<0.001)$, lesion located on the left hemisphere $(\mathrm{OR}=3.11 ; \mathrm{IC} 95 \%, 1.41-6.84$, $\mathrm{p}=0.005)$ and cognitive deterioration $(\mathrm{OR}=3.18$; IC95\%, 1.42-7.10, $\mathrm{p}=0.005)$ were independently associated with DII. Conclusions: Smoking, lesions located on the left hemisphere and cognitive deterioration were associated with DII but further studies are needed to elucidate the nature of these associations.

KEYWORDS: Brain Vascular Accident, Risk Factors, Depression, Cognitive dysfunction. (Source: MeSH NLM). INTRODUCCIÓN

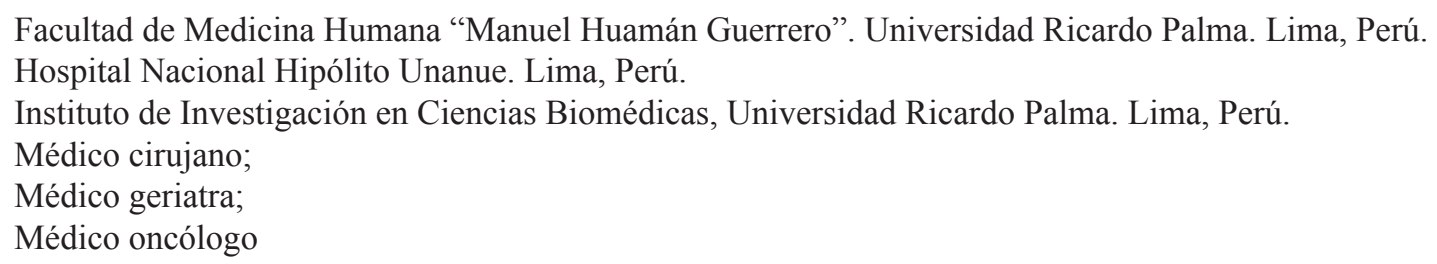


El ictus isquémico (II) es considerada la enfermedad neurológica más frecuente y una de las principales causas de mortalidad a nivel mundial junto con la cardiopatía isquémica. Se estima que en el año 2016, 15,2 millones de personas fallecieron en el mundo como consecuencia del II y a pesar de los esfuerzos dirigidos a su prevención, se calcula que la tasa de incidencia anual de II a nivel mundial es de aproximadamente 200 casos por cada 100000 habitantes por año y la prevalencia de 600 casos por cada 100000 habitantes $(1,2)$. En el Perú, $15 \%$ de todas las muertes prematuras son causadas por el II, siendo la edad promedio para su aparición de 62 a 65 años, con predominio del sexo masculino (3).

La falta de un diagnóstico oportuno del II, la cual puede alcanzar un preocupante $25 \%$ de los casos (4), el desconocimiento de los factores de riesgo o signos de alarma, el elevado costo del tratamiento, la carencia de infraestructura y recursos junto a la falta de un equipo de rehabilitación integral (5), determinan que la mortalidad por II en los hospitales públicos de nuestro país haya alcanzado el 19,7\%, una de las más altas a nivel mundial (6).

Son numerosas las complicaciones que se pueden presentar luego de un II. Usualmente se presta mayor atención al deterioro de la función motora, siendo la rehabilitación y seguimiento del paciente usualmente enfocados en ello, sin embargo, se sabe que también puede existir una afección importante en la cognición, el comportamiento o la afectividad. Dentro de la esfera afectivo-cognitiva, cabe destacar a la depresión, la cual es la complicación neuropsiquiátrica más frecuente y que puede limitar la recuperación funcional, cognitiva, calidad de vida y reinserción social de los pacientes (7).

Según el DSM-V, con el término DPI se hace referencia al episodio depresivo mayor tras un ictus, que incluye síntomas en las áreas afectiva, cognitiva y somática. Para el diagnóstico es necesario tener evidencia clínica y de laboratorio de que la DPI es consecuencia fisiológica directa de la enfermedad vascular cerebral (8). Sin embargo, en estudios previos se han utilizado diferentes escalas para su diagnóstico, como la Hamilton Depression Rating Scale (HDRS) $(9,10)$, Geriatric Depression Scale (GDS) (11), Patient Health Questionnaire-9 (PHQ-9) (12) y Post-stroke Depression Rating Scale (PSDS) (13), herramientas que han demostrado una adecuada sensibilidad y especificidad.

La prevalencia de depresión post ictus isquémico (DPI) varía entre 10-64\%, como consecuencia de diferencias en la metodología, sede y población estudiada (14). En un estudio de 3689 pacientes hospitalizados por ictus isquémico en hospitales de Londres (UK) durante el periodo 1995-2006, se encontró una frecuencia de depresión de 33\%, 28\% y $31 \%$, luego de 3 meses, un año y 5 años después del alta respectivamente. En la misma investigación, los factores predictores de DPI fueron: la severidad del compromiso neurológico, la incapacidad para trabajar y el deterioro cognitivo (15). A pesar de todo lo expuesto, la DPI es por lo general una condición infra diagnosticada e infra tratada.

Ante la falta de trabajos nacionales sobre el tema, se realizó la presente investigación, cuyo objetivo fue determinar los factores de riesgo de DPI, en pacientes adultos atendidos ambulatoriamente, en un hospital de nivel III de Lima.

\section{MATERIAL Y MÉTODOS}

\section{Tipo y diseño de estudio}

Estudio observacional, analítico, de casos y controles no pareado en un grupo de pacientes mayores de 18 años, de ambos sexos, con antecedente de II en los cinco últimos años, atendidos en la consulta externa de Neurología del Hospital Santa Rosa de Lima - Perú, perteneciente al Ministerio de Salud, durante el periodo setiembre - diciembre del 2018. El presente trabajo de investigación se desarrolló en el contexto del IV Curso-Taller de Titulación por Tesis de la Facultad de Medicina Humana "Manuel Huamán Guerrero" de la Universidad Ricardo Palma, según enfoque y metodología publicada (16).

\section{Población y muestra}

La población del estudio estuvo constituida por todos los pacientes mayores de edad, con antecedente de II en los cinco últimos años, que fueron atendidos en la consulta externa del Neurología del Hospital Santa Rosa, durante el periodo de estudio. Se consideró como caso al paciente con el diagnóstico de depresión y como control al paciente sin depresión. Fueron excluidos los pacientes con un compromiso neurológico muy grave al momento de la evaluación (puntaje $\geq 25 \mathrm{en}$ la Escala del NIHSS (National Institute of Health Stroke Scale) (17), deterioro cognitivo severo (puntaje $\geq 7$ en el test de Pfeiffer) (18) y diagnóstico de depresión o enfermedad neurológica previa al II.

Para el cálculo de tamaño muestral, se utilizó el software libre Epi-Info v.5, donde la frecuencia de 
exposición en los controles de la variable a estudiar fue de 0,5 , el nivel de confianza de $95 \%$, la potencia de $80 \%$, Odds Ratio (OR) previsto de 2,5 y una relación de caso - control de 1:1. Se obtuvo una muestra de 81 casos y 81 controles. La muestra se seleccionó por el método del muestreo aleatorio simple.

\section{Variables}

Para el diagnóstico de DPI se utilizó la Escala de Hamilton (Hamilton Depression Rating Scale) en su versión española, considerando al paciente como portador de DPI si el puntaje era $\geq 8$ puntos y $\sin$ DPI si era $<8$ puntos (19). Se registraron las variables clínico - epidemiológicas como: edad $(\leq 65$ y $>65$ años), sexo, antecedente familiar de II (tener uno o más familiares de primer o segundo grado que hayan presentado esta enfermedad), comorbilidades (ninguna, hipertensión arterial, diabetes mellitus o ambas), tabaquismo (consumo $\geq 20$ cigarrillos por día durante al menos cinco años consecutivos), consumo de alcohol (consumo de bebidas alcohólicas al menos una vez por semana durante cinco años consecutivos), tiempo transcurrido desde el II hasta el momento de la entrevista (temprano $\leq 6$ meses y tardío $>6$ meses), hemisferio cerebral afectado y extensión neurológica de la lesión de acuerdo a los informes de la tomografía espiral multicorte (TEM) o resonancia magnética nuclear de cráneo (RMN) (territorio de la circulación cerebral posterior y anterior).

Adicionalmente, se evaluaron el grado de compromiso neurológico mediante la Escala de NIHSS (leve $<4$ puntos, moderado $4-14$, grave 15 - 24 puntos, categorizado para el análisis en leve o moderado vs. grave), la función cognitiva con el Test de Pfeiffer, el cual consta de 10 preguntas y otorga un punto por cada respuesta incorrecta (normal $<4$ puntos, deterioro cognitivo $4-7$ puntos) y el estado funcional en el mes previo al estudio utilizando el Índice de Barthel de actividades básicas de la vida diaria (independiente 100 puntos, dependencia leve 91 - 99 puntos, dependencia moderada $61-90$ puntos, dependencia severa $\leq 60$ puntos, categorizado para el análisis en dependencia leve vs moderada o severa) (20).

\section{Técnicas de recolección y procesamiento de datos}

Luego de la firma del consentimiento informado, se procedió a la toma de datos de las historias clínicas de los pacientes y a una entrevista donde se aplicaron los tests y escalas alusivos al estudio. Para garantizar la confidencialidad de los participantes, las preguntas se respondieron de forma anónima. Luego de la entrevista, las fichas de recolección de datos se colocaron en un sobre cerrado hasta el momento de su digitación.

Los resultados fueron registrados en una base de datos utilizando el programa Microsoft Excel 2013 y procesados con el programa estadístico SPSS v. 25 para Windows. Se utilizó la prueba de Chi Cuadrado o la prueba exacta de Fisher para evaluar las diferencias entre los pacientes con y sin depresión en el caso de variables categóricas, la prueba de t- test de Student en el caso de variables continuas y el test de Mantel y Haenszel para el análisis de tendencias lineares en proporciones para el caso de variables multicategóricas, considerándose un valor de $\mathrm{p}<0,05$ como significativo.

Fueron calculados los OR (odds ratio) y los intervalos de confianza al 95\% (IC) para las variables consideradas factores asociados a depresión. A continuación y para controlar el posible efecto de variables de confusión, se realizó un análisis multivariado de regresión logística, considerándose en el modelo final sólo las variables que en el modelo bivariado alcanzaron un valor de $\mathrm{p}<0,20$.

\section{Aspectos éticos}

El proyecto de investigación fue aprobado por la Oficina de Docencia e Investigación y el Comité de Ética del Hospital Santa Rosa, así como por el Instituto de Investigación en Ciencias Biomédicas (INICIB) y el Comité de Etica en Investigación de la Universidad Ricardo Palma.

\section{RESULTADOS}

Se incluyeron en total de 162 pacientes, $50 \%$ varones, edad media $67,7 \pm 5,49$, rango: $53-82$ años. La edad media de los casos fue 69,3 $\pm 5,1$ y la de los controles $66,1 \pm 5,4$ años $(\mathrm{p}<0,001)$. Las características clínicas y epidemiológicas de los pacientes estudiados son mostradas en la tabla 1.

En el análisis bivariado se encontró asociación entre la variable dependiente DPI y la edad $>65$ años, sexo masculino, antecedente familiar de II, tabaquismo, localización de la lesión en el hemisferio izquierdo, territorio afectado de la circulación cerebral anterior y deterioro cognitivo. Un tiempo transcurrido menor o igual a 6 meses desde la ocurrencia del II, se 
Tabla 1. Características generales de los pacientes estudiados $(\mathrm{N}=162)$.

\begin{tabular}{|c|c|c|c|}
\hline Variables & $\operatorname{Casos}(\mathrm{N}=81)$ & Controles $(\mathrm{N}=\mathbf{8 1})$ & $\mathbf{p}$ \\
\hline Edad & & & 0,041 \\
\hline$\leq 65$ años & $19(23,5 \%)$ & $31(38,3 \%)$ & \\
\hline$>65$ años & $62(76,5 \%)$ & $50(61,7 \%)$ & \\
\hline Sexo & & & 0,008 \\
\hline Femenino & $32(39,5 \%)$ & $49(60,5 \%)$ & \\
\hline Masculino & $49(60,5 \%)$ & $32(39,5 \%)$ & \\
\hline Antecedente familiar de $\mathrm{II}^{\circ}$ & & & 0,001 \\
\hline No & $40(49,4 \%)$ & $60(74,1 \%)$ & \\
\hline Sí & $41(50,6 \%)$ & $21(25,9 \%)$ & \\
\hline Comorbilidades & & & 0,226 \\
\hline Ninguna & $29(35,8 \%)$ & $40(49,4 \%)$ & \\
\hline Hipertensión arterial & $18(22,2 \%)$ & $18(22,2 \%)$ & \\
\hline Diabetes mellitus & $16(19,8 \%)$ & $13(16 \%)$ & \\
\hline Ambas & $18(22,2 \%)$ & $10(12,3 \%)$ & \\
\hline Tabaquismo & & & $<0,001$ \\
\hline No & $36(44,4 \%)$ & $60(74,1 \%)$ & \\
\hline Sí & $45(55,6 \%)$ & $21(25,9 \%)$ & \\
\hline Consumo de alcohol & & & 0,345 \\
\hline No & $40(49,4 \%)$ & $46(56,8 \%)$ & \\
\hline Sí & $41(50,6 \%)$ & $35(43,2 \%)$ & \\
\hline Tiempo transcurrido desde II & & & $<0,001$ \\
\hline Tardío (> 6 meses) & $67(82,7 \%)$ & $31(38,3 \%)$ & \\
\hline Temprano ( $\leq 6$ meses $)$ & $14(17,3 \%)$ & $50(61,7 \%)$ & \\
\hline Hemisferio afectado & & & 0,001 \\
\hline Derecho & $25(30,9 \%)$ & $47(58 \%)$ & \\
\hline Izquierdo & $56(69,1 \%)$ & $34(42 \%)$ & \\
\hline Extensión de la lesión & & & 0,040 \\
\hline Circulación cerebral posterior & $13(16 \%)$ & $24(29,6 \%)$ & \\
\hline Circulación cerebral anterior & $68(84 \%)$ & $57(70,4 \%)$ & \\
\hline Compromiso neurológico & & & $<0,001$ \\
\hline Leve & $3(3,7 \%)$ & $32(39,5 \%)$ & \\
\hline Moderado & $56(69,1 \%)$ & $37(45,7 \%)$ & \\
\hline Grave & $22(27,2 \%)$ & $12(14,8 \%)$ & \\
\hline Deterioro cognitivo & & & 0,007 \\
\hline No & $34(41,9 \%)$ & $51(63 \%)$ & \\
\hline Sí & $47(58,1 \%)$ & $30(37 \%)$ & \\
\hline Estado funcional & & & 0,395 \\
\hline Dependencia leve & $20(24,7 \%)$ & $29(35,8 \%)$ & \\
\hline Dependencia moderada & $51(63 \%)$ & $43(53,1 \%)$ & \\
\hline Dependencia severa & $10(12,3 \%)$ & $9(11,1 \%)$ & \\
\hline
\end{tabular}


Tabla 2. Modelo bivariado de los factores de riesgo de depresión post ictus isquémico (DPI) $(\mathrm{N}=162)$.

\begin{tabular}{|c|c|c|}
\hline Variables & OR crudo (IC95\%) & $\mathbf{p}$ \\
\hline \multicolumn{3}{|l|}{ Edad } \\
\hline$\leq 65$ años & 1 & \\
\hline$>65$ años & $2,02(1,02-4,00)$ & 0,041 \\
\hline \multicolumn{3}{|l|}{ Sexo } \\
\hline Femenino & 1 & \\
\hline Masculino & $2,34(1,24-4,40)$ & 0,008 \\
\hline \multicolumn{3}{|l|}{ Antecedente familiar de $\mathrm{II}^{\circ}$} \\
\hline No & 1 & \\
\hline Sí & $2,92(1,51-5,67)$ & 0,001 \\
\hline \multicolumn{3}{|l|}{ Comorbilidades } \\
\hline Ninguna & 1 & \\
\hline Hipertensión arterial & $0,55(0,20-1,52)$ & 0,255 \\
\hline Diabetes mellitus & $0,68(0,23-1,98)$ & 0,684 \\
\hline Ambas & $0,40(0,16-1,00)$ & 0,050 \\
\hline \multicolumn{3}{|l|}{ Tabaquismo } \\
\hline No & 1 & \\
\hline Sí & $3,57(1,84-6,92)$ & $<0,001$ \\
\hline \multicolumn{3}{|l|}{ Consumo de alcohol } \\
\hline No & 1 & \\
\hline Sí & $1,34(0,72-2,50)$ & 0,345 \\
\hline \multicolumn{3}{|l|}{ Tiempo transcurrido desde II } \\
\hline Tardío (> 6 meses) & 1 & \\
\hline Temprano ( $\leq 6$ meses) & $0,13(0,06-0,26)$ & $<0,001$ \\
\hline \multicolumn{3}{|l|}{ Hemisferio afectado } \\
\hline Derecho & 1 & \\
\hline Izquierdo & $3,09(1,62-5,90)$ & 0,001 \\
\hline \multicolumn{3}{|l|}{ Extensión de la lesión } \\
\hline Circulación cerebral posterior & 1 & \\
\hline Circulación cerebral anterior & $2,20(1,02-4,71)$ & 0,042 \\
\hline \multicolumn{3}{|l|}{ Compromiso neurológico } \\
\hline Leve - Moderado & 1 & \\
\hline Grave & $2,14(0,97-4,69)$ & 0,057 \\
\hline \multicolumn{3}{|l|}{ Deterioro cognitivo } \\
\hline No & 1 & \\
\hline Sí & $2,35(1,25-4,41)$ & 0,007 \\
\hline \multicolumn{3}{|l|}{ Estado funcional } \\
\hline Dependencia leve & 1 & \\
\hline Dependencia moderada-severa & $1,59(0,81-3,12)$ & 0,174 \\
\hline
\end{tabular}


Tabla 3. Modelo multivariado de los factores de riesgo de depresión post ictus isquémico (DPI) $(\mathrm{N}=162)^{*}$.

\begin{tabular}{lcc}
\hline Variables & OR ajustado $(\mathbf{I C 9 5 \% )}$ & $\mathbf{P}$ \\
\hline Tabaquismo & $4,67(1,99-10,92)$ & $<0,001$ \\
Hemisferio afectado & & \\
Izquierdo & $3,11(1,41-6,84)$ & 0,005 \\
Deterioro cognitivo & $3,18(1,42-7,10)$ & 0,005 \\
\hline * se incluyeron las variables con un valor $\mathrm{p}<0,20$ en el modelo bivariado
\end{tabular}

asoció a un menor riesgo de DPI (OR $=0,13$; IC95\%, $0,06-0,26, p<0,001)$. No se evidenciaron diferencias significativas entre los grupos de casos y controles respecto a comorbilidades, consumo de alcohol, compromiso neurológico y estado funcional (tabla 2).

Con las variables que demostraron un valor de $\mathrm{p}<$ 0,20 en el modelo bivariado, se construyó un modelo multivariado de regresión logística, encontrándose finalmente asociación entre DPI y tabaquismo $(\mathrm{OR}=$ 4,67; IC95\%, 1,99 - 10,92, p < 0,001), localización de la lesión en el hemisferio izquierdo $(\mathrm{OR}=3,11$; y deterioro cognitivo (OR = 3,18; IC95\%, 1,42-7,10, p $=0,005)($ tabla 3 ).

\section{DISCUSIÓN}

El área afectiva usualmente no es considerada en la evaluación de rutina de los pacientes con II. El diagnóstico de DPI constituye todo un reto para el médico que labora en consulta externa, donde el tiempo y los recursos son usualmente escasos. Para el diagnóstico de DPI nosotros empleamos la HDRS, instrumento validado previamente en los pacientes con ictus, con una sensibilidad de $73,1 \%$, especificidad de $81,6 \%$ y coeficiente alfa de Cronbach $>0,80$ para el diagnóstico de DPI (puntaje en el test $>7$ puntos) (21).

En nuestra investigación, los pacientes con tabaquismo tuvieron mayor probabilidad de presentar DPI en comparación a los que no lo tuvieron. Este no ha sido un factor de riesgo evaluado en estudios previos, sin embargo, cabe resaltar que el tabaco propicia la hipercoagulabilidad, eleva la presión arterial y lesiona el endotelio, aumentando el riesgo de un II que pueda interrumpir los circuitos cortico-estriado-pálidotálamo-corticales resultando en síntomas depresivos (22). Asimismo, Fluharty et al. (23), en una revisión sistemática de 148 estudios, plantean una asociación bidireccional entre el tabaquismo con depresión y ansiedad, siendo necesarios más estudios para demostrar una posible relación de causalidad.
Los pacientes que presentaron lesiones isquémicas del hemisferio cerebral izquierdo, tuvieron un mayor riesgo de presentar DPI, resultado similar a otros estudios, donde se describe que las lesiones en las regiones corticales frontal y dorsolateral izquierdas, en el lóbulo frontal izquierdo y ganglios basales izquierdos podrían interrumpir los circuitos de las monoaminas encargadas del tono del humor como la serotonina, conduciendo así a la depresión (10). Otros mecanismos propuestos que podrían contribuir a la aparición de DPI son: una disfunción glutaminérgica, hipercortisolismo e inflamación isquémica crónica (24).

La naturaleza de la asociación entre la DPI y el deterioro cognitivo es compleja. El deterioro cognitivo luego de un II es un bien documentado factor pronóstico de DPI (15), habiéndose propuesto que las secuelas del ictus como afasia, discapacidad funcional, pérdida laboral y aislamiento social, podrían condicionar la aparición de humor depresivo (25). Se sabe también que la amígdala está involucrada en la regulación del humor y las emociones. En el Sidney Stroke Study, se demostró que aquellos pacientes que habían sufrido un II o ataque isquémico transitorio, presentaban una amígdala de menor tamaño, especialmente aquellos con síntomas cognitivos (26). Por otro lado, se ha descrito que la depresión de inicio tardío podría ser un pródromo antes que un factor de riesgo para el desarrollo de una demencia (27).

La DPI suele aparecer en los primeros meses después del II. En un estudio prospectivo de 100 pacientes con el diagnóstico de II, se observó una frecuencia de DPI de $81 \%$ al inicio de la evaluación y luego de 5 meses la cifra descendió a 65\% (28). Por el contrario, en nuestro estudio, el riesgo de DPI fue menor para los pacientes con menos de 6 meses desde la ocurrencia del II. Es probable que con un tiempo de observación más largo, pudiera evidenciarse el verdadero impacto que tienen las secuelas del ictus en 
la aparición de la DPI.

En trabajos previos se ha encontrado un mayor riesgo de DPI en pacientes de edad avanzada, sexo masculino, con antecedente familiar de II, comorbilidades, alcoholismo, lesión en el territorio de la arteria cerebral media, compromiso neurológico severo y dependencia funcional $(7,14,15,24$, 28). Nosotros sin embargo no encontramos dichas asociaciones.

Esta investigación representa una primera aproximación al estudio de la DPI en nuestro país. Dentro de sus limitaciones están el limitado tamaño muestral; la inclusión de pacientes atendidos en un consultorio especializado de un hospital público de III nivel de atención, lo cual pudiera comprometer su validez externa; así como la falta de una entrevista psiquiátrica formal en el protocolo de evaluación, la cual podría haber servido para distinguir mejor entre una DPI y episodios depresivos secundarios a eventos estresantes en la vida del paciente.

Nuestros resultados permiten concluir que el tabaquismo, la localización de la lesión en el hemisferio cerebral izquierdo y el deterioro cognitivo, fueron factores independientemente asociados al desarrollo de DPI. La evaluación de la esfera cognitiva - afectiva debería formar parte del manejo multidisciplinario de los pacientes con II.

\section{Declaración de financiamiento y de conflicto de intereses:}

Este trabajo fue financiado por los autores. Los autores declaran no tener conflictos de intereses.

\section{Contribución de autoría:}

MH y JL fueron responsables de la concepción y diseño de estudio. MH fue responsable de la recolección de datos. MH, JL y JAD fueron responsables del análisis e interpretación de los resultados así como de la redacción del artículo: todos los autores realizaron la revisión crítica y aprobación final del mismo.

\section{Correspondencia:}

Jaime Enrique Lama Valdivia

Calle Pirandello 488, Dpto. 201, Urb. San Borja.

Lima 41, Perú.

Teléfono: 051999093383
Correo electrónico: jlamav@hotmail.com

\section{REFERENCIAS BIBLIOGRÁFICAS}

1. World Health Organization. Las 10 principales causas de defunción. Washington DC: World Health Organizatio; 2018. (Citado 1 septiembre de 2018) Disponible en: http://www.who.int/es/news-room/ fact-sheets/detail/the-top-10-causes-of-death

2. INFOMED. Factográfico de Salud: Accidente Cerebrovascular. Estadísticas Mundiales. La Habana, Cuba: Infomed; 2017 (Citado 1 septiembre de 2018) Disponible en: http://www.sld.cu/anuncio/2018/01/ 10/factografico-de-salud-accidente-cerebrovascularestadisticas-mundiales

3. Malaga G, Saldaña T, Busta-Flores P, Carbajal A, Santiago-Mariaca K. La enfermedad cerebrovascular en el Perú: estado actual y perspectivas de investigación clínica. Acta Med Perú. 2018; 35(1):51-

4. Davalos L, Málaga G. El accidente cerebrovascular en el Perú: una enfermedad prevalente olvidada y desatendida. Rev Peru Med Exp Salud Publica. 2014; 31(2):400-1.

5. Ferri C P, Schoenborn C, Kalra L, et al. Prevalence of stroke and related burden among older people living in Latin America, India and China. J Neurol Neurosurg Psychiatry. 2011; 82(10):1074-82.

6. Castañeda-Guarderas A, Beltrán-Ale G, CasmaBustamante R, Ruiz-Grosso P, Málaga G. Registro de pacientes con accidente cerebro vascular en un hospital público del Perú, 2000-2009. Rev Peru Med Exp Salud Publica. 2011; 28(4):623-7.

7. Espárrago G, Castilla-Guerra L, Fernandez M, Ruiz $\mathrm{S}$, Jiménez M. Depresión post ictus: una actualización. Neurología. 2015; 30(1):23-31.

8. American Psychiatric Association. Diagnostic and statistical manual of mental disorders. 5th ed. Arlingon, VA: American Psychiatric Publishing; 2013.

9. Hamilton M. A rating scale for depression. J Neurol Neurosurg Psychiatry. 1960; 23:56-62.

10. Tu J, Wang L-X, Wen H-F, Xu Y-C, Wang P-F. The association of different types of cerebral infarction with post-stroke depression and cognitive impairment. Medicine (Baltimore). 2018; 97(23):e10919. doi: 10.1097/MD.0000000000010919

11. Johnson G, Burvill P, Anderson C, Jamrozik K, Stewart-Wynne E, Chakera T. Screening instruments for depression and anxiety following stroke: experience in the Perth community stroke study. Acta Psychiatr Scand. 2007; 91:252-7.

12. De-Man-van Ginkel JM, Hafsteinsdòttir T, et al. An efficient way to detect poststroke depression by subsequent administration of a 9-item and a 2-item patient health questionnaire. Stroke. 2012; 43:854- 
856.

13. Quaranta D, Marra C, Gainotti G. Mood disorders after stroke: diagnostic validation of the poststroke depression rating scale. Cerebrovasc Dis. 2008; 26:237- 43.

14. Concepción OF, Fleita MM, Barrero YM, Velázquez $\mathrm{N}$ de JS, Rojas Fuentes JO. Depresión post-ictus: frecuencia y factores determinantes. RCNN. 2012; 2(1):9-16.

15. Ayerbe L, Ayis S, Rudd AG, Heuschmann PU, Wolfe CD. Natural history, predictors, and associations of depression 5 years after stroke: the South London Stroke Register. Stroke. 2011; 42(7):1907-11.

16. De-la-Cruz-Vargas JA, Correa-Lopez LE, AlatristaGutierrez-de-Bambaren M, Sanchez HH. Promoviendo la investigación en estudiantes de Medicina y elevando la producción científica en las universidades: experiencia del Curso Taller de Titulación por Tesis. Educación Médica. 2019; 20(4):199-205. DOI: https://doi.org/10.1016/j. edumed.2018.06.003

17. Montaner J, Alvarez-Sabin J. La escala de ictus del National Institute of Health (NIHSS) y su adaptación al español. Neurología. 2006; 21(4):192-202.

18. Martínez J, Dueñas R, Onis Mc, Aguado C, Albert $\mathrm{C}$, Luque R. Adaptación y validación al castellano del cuestionario de Pfeiffer (SPMSQ) para detectar la existencia de deterioro cognitivo en mayores de 65 años. Med Clin (Barc). 2001; 117(4):129-134.

19. Ramos-Brieva J. Validación de la versión castellana de la escala de Hamilton para la depresión. Actas Luso-Esp Neurol Psiquiatr. 1986; 14:324-334.

20. Baztán JJ, Pérez del Molino J, Alarcón T. Índice de Barthel: instrumento válido para la valoración funcional de pacientes con enfermedad cerebrovascular. Rev Esp Geriatr Gerontol. 1993; 28:32-40.

21. Aben I, Verhey F, Lousberg R, Lodder J, Honig A. Validity of the Beck depression inventory, Hospital anxiety and Depression scale, SCL-90 and Hamilton depression rating scale as screening instruments for depression in stroke patients. Psychosomatics. 2002; 43:386-393.

22. Gonzáles R, Landínez D. Epidemiología, etiología y clasificación de la enfermedad vascular cerebral. Arch Med (Manizales). 2016; 16(2):495-507.

23. Fluharty M, Taylor AE, Grabski M, Munafó MR. The association of cigarette smoking with depression and anxiety: a systematic review. Nicotine Tob Res. 2017; 19(1):3-13.

24. Feng C, Fang M, Liu X-Y. The Neurobiological Pathogenesis of Poststroke Depression. Sci. World J. 2014; 97(23):0-0. DOI: http://dx.doi. org/10.1155/2014/521349

25. Kauhanen M, Korpelainen JT, Hiltunen P, et al. Poststroke depression correlates with cognitive impairment and neurological deficits. Stroke. 1999; 30:1875-80.

26. Sachdev PS, Chen X, Joscelyne A, Wen W, Brodaty $\mathrm{H}$. Amygdala in stroke/transient ischemic attack patients and its Relationship to cognitive impairment and psychopathology: the Sydney Stroke Study. Am J Geriatr Psychiatry. 2007; 15:487-496.

27. Brommelhoff JA, Gatz M, Johansson B, McArdle JJ, Fratiglioni L, Pedersen NL. Depression as a risk factor or prodromal feature for dementia? Findings in a population-based sample of Swedish twins. Psychol Aging. 2009; 24(2):373-84.

28. Vojtikiv-Samoilovska D, Arsovska A. Prevalence and Predictors of Depression after Stroke - Results from a Prospective Study. Open Access Maced J Med Sci. 2018; 6 (5):824-8.

Recibido: $14 / 01 / 2020$

Aceptado: 25/06/2020 